\title{
EFFECT OF SILICON DIOXIDE ENRICHED WATER DURING CHRONIC INGESTION OF ALUMINUM ON FUNCTIONAL CHARACTERISTICS OF PERITONEAL MACROPHAGES
}

\author{
Tanja Dzopalic ${ }^{*}$, Dragana Zmijanjac², Adil Ehmedah², Boris Djindjic1, Dejan Krstic3, \\ Marija Dakovic-Bjelakovic ${ }^{1}$, Biljana Bozic-Nedeljkovic ${ }^{2}$
}

\author{
${ }^{1}$ Medical Faculty, University of Nis, Nis, Serbia \\ ${ }^{2}$ Faculty of Biology, University of Belgrade, Belgrade, Serbia \\ 3 Faculty of Occupational Safety, University of Nis, Nis, Serbia
}

\begin{abstract}
Background: Silicon dioxide (SiO2) is an oligomineral found naturally in water, plants, and animals. One of the most important sources of this mineral is drinking water, where certain amount of dissolved amorphous silicon dioxide can be detected. Lack of $\mathrm{SiO}_{2}$ is associated with moderate disorders in the metabolism of bone tissue. Aluminum food intake enhances inflammation and the production of inflammatory cytokines. It is shown that silicon dioxide has an antagonistic effect with the aluminum in the body and may have a preventive role in numerous diseases. Therefore, the application of natural protectors that have the ability to reduce inflammatory responses and other harmful effects of aluminum are of special importance for contemporary nutrition. Aim: The main goal of this study was to evaluate the effect of silicon dioxide enriched water during chronic ingestion of aluminum on functional characteristics of peritoneal macrophages as one of the best-studied macrophage population. Materials and Methods: We used the model of chronic intoxication with aluminum for a period of 90 days by gavages with a solution of aluminum chloride in the form of aluminum salt dissolved in distilled water (1.6 mg/ $\mathrm{kg}$ in $0.5 \mathrm{ml}$ daily water intake). In total, 21 female Wistar Albino rats were divided into 3 groups, each consisting of 7 animals: chronically intoxicated with aluminum [non-treated (NT) and per os treated (T) with water enriched with silicon dioxide (2O mg/l)] and sham-intoxicated (S). PMF (LPS- or PMA-stimulated) were evaluated according to phagocytic activity and metabolic activity after $24 \mathrm{~h}$ cultivation in vitro. The study also included evaluation of supernatant cytokine (TNF- $\alpha$, soluble (s) ICAM-1) concentration in supernatants by ELISA method. Results: PMF from animals chronically intoxicated with aluminum (NT) showed lower metabolic viability/activity compared with PMF isolated from sham animals (S). Treatment of intoxicated animals with water enriched with SiO2 partially restored metabolic viability. T-PMF had higher metabolic viability compared to NT-PMF but still significantly lower than S-PMF. PMF of aluminumintoxicated animals (NT-PMF) showed very low phagocytic activity, whereas SiO2 enriched water significantly upregulated this activity. A daily intake of aluminum increased the release of TNF- $\alpha$, while the SiO2 treatment reduced these levels. Up-regulated ICAM-1 shredding by T-PMF were followed by the production of TNF- $\alpha$. Conclusion: Our study showed that treatment with water enriched with SiO2 in concentration of 20 mg/L partially normalized characteristic of PMF of animals intoxicated with aluminum. We can suggest that SiO2 could be a natural antidote of the aluminum and we may presume its possible beneficial role in decreasing the aluminum toxicity commonly present in water and food.
\end{abstract}

Key words: Aluminum, silicon dioxide, peritoneal macrophages, inflammation

DOI: $10.21175 /$ RadProc.2016.35

\section{INTRODUCTION}

Silicon dioxide (SiO2), also known as silica, is an oligomineral found naturally in water, plants, and animals. Along with being found pretty much everywhere on Earth, $\mathrm{SiO} 2$ is found in small quantities in many types of food and supplements. One of the most important sources of this mineral is drinking water where certain amount of dissolved amorphous silicon dioxide can be detected [1]. According to The Food and Drug Administration (FDA), there is no evidence that $\mathrm{SiO} 2$ can affect reproductive health, birth weight, or body weight. FDA also described $\mathrm{SiO} 2$ as a safe food additive, as do the World Health
Organization (WHO) and the European Food Safety Authority (EFSA). Silicon-deficiency studies in growing animals in the 1970 s and 1980s reported stunted growth and profound defects in bone and other connective tissues in these animals [2],[3]. However, more recent studies have found mild alterations in bone metabolism without any adverse health effects, thus the biological role of silicon remains unknown [4],[5].

Aluminum ( $\mathrm{Al}$ ) is ubiquitous and is the most abundant metal element in our environment [6], [7] A main route of exposure to aluminum is through food and water. For many years, exposure to aluminum in the form of hydroxide was suggested to favor an abnormal immune response in different diseases,

\footnotetext{
tanjche80@gmail.com
} 
including autoimmune conditions, inflammatory bowel disease and breast cancer [8]-[10]. Some studies described an antagonistic effect of $\mathrm{SiO}_{2}$ and $\mathrm{Al}$, thus indicating that silica could have preventive role in numerous diseases [11]. Therefore, the applications of natural protectors that have the ability to reduce harmful effects of aluminum are of special importance for contemporary nutrition.

Peritoneal macrophages (PMF) from experimental animals are an exceptionally powerful model system to investigate macrophage phenotypes and functions in response to specific stimuli, resembling as much as possible the conditions observed in various pathophysiological settings such as intoxication with aluminum. A number of animal experiments proved that $\mathrm{Al}$ exposure caused inhibitory effects on the immune function of different immune cells including macrophages [12]. Also, there are data showing that capture of crystalline silica by macrophages can modify their metabolic activity [13],[14].

Having that in mind, main goal of our study was to evaluate the effect of water enriched in soluble $\mathrm{SiO}_{2}$ during chronic ingestion of aluminum on functional characteristics of peritoneal macrophages as a one of the best-studied macrophage population.

\section{MATERIALS AND METHODS}

We used model of chronic intoxication with aluminum for a period of 90 days by gavages with a solution of aluminum chloride in the form of aluminum salt dissolved in distilled water $(1.6 \mathrm{mg} / \mathrm{kg}$ in $0.5 \mathrm{~mL}$ daily water intake). In total, 21 female Wistar Albino rats, 2 months old, were divided into 3 groups, each consisting of 7 animals: chronically intoxicated with a soluble aluminum [non-treated (NT) and per os treated (T) with water enriched in soluble form of silicon dioxide (20 mg/l)] and sham-intoxicated (S). After 90 days of treatment, the animals were anesthetized by Ketamin $\mathrm{HCl}$ (50 mg/kg).

\subsection{Isolation of peritoneal cells}

Peritoneal cells were harvested by peritoneal lavage with $20 \mathrm{ml}$ of ice cold PBS/NaEDTA. Harvested cells were purified using 40\% OptiPrep gradient. The purity of peritoneal macrophage population obtained by this method was $85 \%$, according to staining with FITCconjugated anti-CD68 antibodies (AbD Serotec,Oxford, UK). After that, cells were seeded in 96 well plates, $10^{5}$ cells per well and cultured in standard conditions (5\%CO2, 37oC). Parallel set of cultures was placed where macrophages were stimulated by lipopolysaccharide (LPS) $(10 \mu \mathrm{g} / \mu \mathrm{l})$. Phagocytic activity, metabolic activity, nitric-oxide (NO) production and cytokine releasing by PMF were measured after $24 \mathrm{~h}$ cultivation in vitro.

\subsection{MTT assay}

Metabolic activity was measured by MTT assay. Briefly, MTT (Invitrogen) was added in final concentration $0.5 \mathrm{mg} / \mathrm{ml}$ and incubated for $4 \mathrm{~h}$. Formed formazan crystals were dissolved by adding SDS-HCl (100ul/well). Optical density was measured at $570 / 650 \mathrm{~nm}$ using an ELISA plate reader. All determinations were conducted in quadruplicate. 148

\subsection{Phagocytosis assay}

Phagocytsis assay was carried out according to the technique described by Chen et al. [15]. Supernatants were collected and 50 $\mu$ / well of neutral red (1:300) was added, and incubated for $4 \mathrm{~h}$. The supernatant was discarded and cells were washed three times with PBS. After that, $100 \mu \mathrm{l} /$ well of cell lysing solution (ethanol and $1 \%$ acetic acid at the ratio of $1: 1$ ) was added. The absorbance was measured at 540/650 $\mathrm{nm}$ using an ELISA reader. All determinations were conducted in quadruplicate.

\subsection{NO production}

Nitric oxide (NO), quantified by the accumulation of nitrite as a stable end-product, was determined by a Greiss reaction assay. Briefly, 50- $\mu \mathrm{l}$ samples supernatans were removed from the PMF culture and incubated with an equal volume of Griess reagent [0.35\% 4-aminophenyl sulfone (Sigma-Aldrich, Germany), $\quad 0.1 \% \quad \mathrm{~N}$-(1-naphthyl)ethylenediamine dihydrochloride in $1 \mathrm{M} \mathrm{HCl}$ (POCh, Poland)] at room temperature for $10 \mathrm{~min}$. The absorbance at 540/650 $\mathrm{nm}$ was measured and the concentration of nitrite was calculated with a help of a sodium nitrite standard curve.

\subsection{Detection of cytokines}

Evaluation of cytokines (TNF- $\alpha$, IL-10, soluble (s) ICAM-1) released by PMF was done by detecting their concentration in supernatants by ELISA method according to the manufactures instruction (R\&D Systems, Minneapolis, USA).

\subsection{Statistical analysis}

The differences in parameters between experimental and control groups were evaluated using Student's t-test or one-way ANOVA. Values of $\mathrm{p}<0.05$ or less were considered as statistically significant.

\section{RESULTS AND DISCUSSION}

Macrophages are resident cells found in almost all tissues of the body with numerous functions such as promotion of innate immune response and enhancement of organism's defense capabilities by phagocytosis and secreting cytokines [16-18]. Peritoneal macrophages play key roles in the control of infections, different environmental negative influence and different inflammatory pathologies, as well as in the maintenance of immune response robustness and homeostasis [19], [20].

It is known that chronic aluminum ingestion by water causes macrophages dysfunction in vivo [21]. Results obtained in this study showed that treatment with water highly enriched in $\mathrm{SiO} 2$ decreases the effects of $\mathrm{Al}$ exposure and partially restores functional characteristics of PMF measured by their metabolic activity, phagocytosis capacity, NO and TNF-a production.

Metabolic activity and viability of the cells are very important for their normal functioning and it is one of the first cell characteristics to check when we want to investigate the effect of certain substance. PMF from 
animals chronically intoxicated with aluminum (NT) showed lower metabolic viability/activity compared with PMF isolated from sham animals (S) (see Fig. 1). Our results are in accordance with literature data which show that aluminum suppress macrophage function both in vitro and in vivo [12],[22]-[25] Treatment of intoxicated animals with water enriched in $\mathrm{SiO}_{2}$ partially restores metabolic viability. T-PMF have higher metabolic viability compared to NT-PMF but still significantly lower than S-PMF.

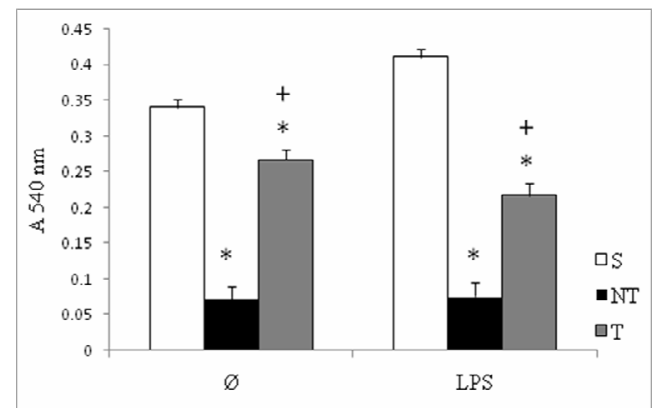

Figure 1. Metabolic activity of tested PMF Metabolic activity was measured by MTT assay. Briefly, MTT was added in final concentration $0.5 \mathrm{mg} / \mathrm{ml}$ and incubated for 4h. Formed formazan crystals were dissolved by adding SDS$\mathrm{HCl}(100 \mu \mathrm{l} /$ well). Optical density was measured at $540 \mathrm{~nm}$ using an ELISA reader. S- Sham, NT- Al intoxicated, nontreated, T-Al intoxicated, treated with water enriched in $\mathrm{SiO}_{2}$

Values are means \pm S.E. $(n=3),{ }^{*} \mathrm{P}<0.05$ vs. $\mathrm{S},+<0.05$ vs.

$$
\text { NT }
$$

Phagocytosis is important function of macrophages. Phagocytosis of pathogens by macrophages initiates the innate immune response, which in turn orchestrates the adaptive response [26]. PMF of aluminum-intoxicated animals (NT-PMF) showed very low phagocytic activity as shown in Figure 2. Other researchers [12] also showed that $\mathrm{Al}$ exposure decreases macrophage phagocytosis capacity. On the other hand, treatment of such intoxicated animals with water enriched with silicon dioxide significantly upregulated their PMF phagocytic activity. Slight increase of this characteristic in $\mathrm{T}$ group is in agreement with the fact that $\mathrm{SiO}_{2}$ stimulates phagocytic activity of PMF against BCG strain mycobacteria [27].

Activation of macrophages is also related with the increased level of NO production. This molecule has microbicide potential and can act as a messenger in inflammation [28]. Unless stimulated, PMF produce very low, undetectable level of NO in all groups (data not shown). When stimulated, S-PMF and T-PMF start to produce certain level of NO, but this is not a note for NT-PMF (see Fig.3). These results are expected because Al also caused suppression of other macrophage function (metabolic activity, phagocytosis, etc.) in our study. Detected increase of NO production by T-PMF compared to NT-PMF may be consequence of $\mathrm{SiO}_{2}$ induced activation of PMF. Literature data show that silica nanoparticles may induce proinflammatory response of PMF [29].

A daily intake of aluminum also affects the release of proinflammatory cytokines such as TNF- $\alpha$ (see Fig. 4). TNF- $\alpha$ is a cytokine involved in inflammation and is produced mainly by activated macrophages. It plays a critical role in a pathophysiology of various diseases, including inflammatory bowel disease [30],
Alzheimer's disease [31], cancer [32]. On the other hand, recent studies are indicating a possible involvement of aluminum in development of these diseases. Namely, de Chambrun et al. [9] have shown deleterious effects of aluminum on intestinal inflammation and mucosal repair suggesting it as an inflammatory bowel diseases risk factor. In addition, Mannello and Canale showed an involvement of aluminum ions in oxidative and inflammatory status perturbations of breast cancer microenvironment [10]. A 15-Year Follow-up of the PAQUID Cohort study pointed that high daily intake of aluminum was significantly associated with increased risk of dementia [11]. Our finding of decreased levels of TNF- $\alpha$ production by NT-PMF correlates with lower metabolic activity of these cells. Also it is consistent with other researchers' data that showed that $\mathrm{Al}$ exposure leads to decrease of TNF- $\alpha$ mRNA expression in PMF and serum TNF- $\alpha$ level. However, an important finding of our study refers to normalizing TNF- $\alpha$ production by PMF isolated from $\mathrm{T}$ animals. This finding can be explained with the results of Park and Park [29]. They showed that treatment with silica increases the level of IL-1, IL-6, TNF- $\alpha$, iNOS, and COX-2 mRNA expression and it may trigger pro-inflammatory responses both in vivo and in vitro.

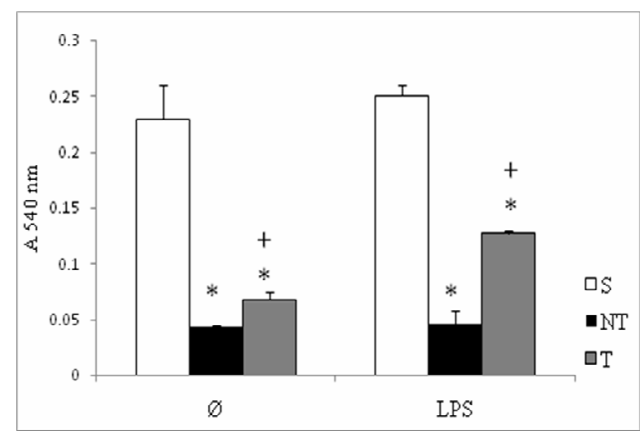

Figure 2. Phagocytic activity of tested PMF

Phagocytotic/pinocityc activity was assessed by Neutral red uptake. Cells were placed in a 96-well plate and cultured at 37 ${ }^{\circ} \mathrm{C}, 5 \% \mathrm{CO} 2$ for $72 \mathrm{~h}$. Culture media were removed and 100 $\mu \mathrm{L} /$ well of neutral red added, and incubated for $4 \mathrm{~h}$. Media were discarded, and macrophages were washed for three times with PBS ( $\mathrm{pH}$ 7.2-7.4).Washed macrophages were resuspended in $100 \mu \mathrm{L} /$ well of cell lysing solution and cultured for $2 \mathrm{~h}$. The phagocytic level was measured as the absorbance at $540 \mathrm{~nm}$. S- Sham, NT- Al intoxicated, nontreated, T-Al intoxicated, treated with water enriched in $\mathrm{SiO}_{2}$

Values are means \pm S.E. $(n=3),{ }^{*} \mathrm{P}<0.05$ vs. $\mathrm{S},+<0.05$ vs. NT

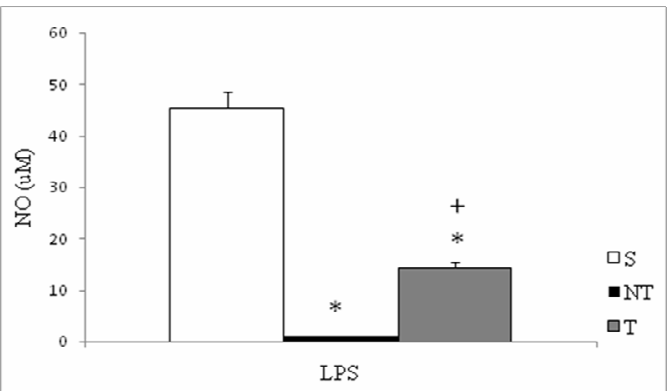

Figure 3. Production of NO by tested PMF Production of NO was measured by Greiss reaction. Briefly, $50 \mathrm{ul}$ of supernatans was mixed with $50 u l$ og Greiss reagens. Optical density was measured after $10 \mathrm{~min}$ at $540 \mathrm{~nm}$ using 
an ELISA reader. S- Sham, NT- Al intoxicated, non-treated, $\mathrm{T}$-Al intoxicated, treated with water enriched in $\mathrm{SiO} 2$ Values are means \pm S.E. $(n=3),{ }^{*} \mathrm{P}<0.05$ vs. $\mathrm{S},+<0.05$ vs. NT

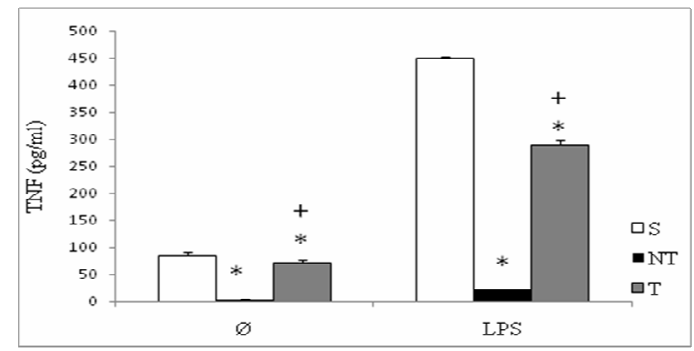

Figure 4. Production of TNF- $\alpha$ by tested PMF

Cytokine concentration of TNF- $\alpha$ was measured in cell supernatants by ELISA method according to the manufacturer's instructions. S- Sham, NT- Al intoxicated, non-treated, T-Al intoxicated, treated with water enriched in $\mathrm{SiO} 2$

Values are means \pm S.E. $(n=3),{ }^{*} \mathrm{P}<0.05$ vs. $\mathrm{S},+<0.05$ vs. NT

Beyond its classically described functions as an adhesion molecule, ICAM-1 has now been characterized as possessing a role in signal transduction. Such function of ICAM-1 seems to be associated with pro-inflammatory pathways. In particular, ICAM-1 signaling seems to produce a recruitment of inflammatory macrophages [33], [34]. Up-regulated ICAM-1 shredding by T-PMF in our study (see Fig. 5) are in agreement with studies where it follows the production of pro-inflammatory TNF- $\alpha$ [35]. Additionally, Hubbard and Giardina showed that silica increases ICAM-1 expression significantly by $48 \mathrm{~h}$ of in vitro stimulation [36].

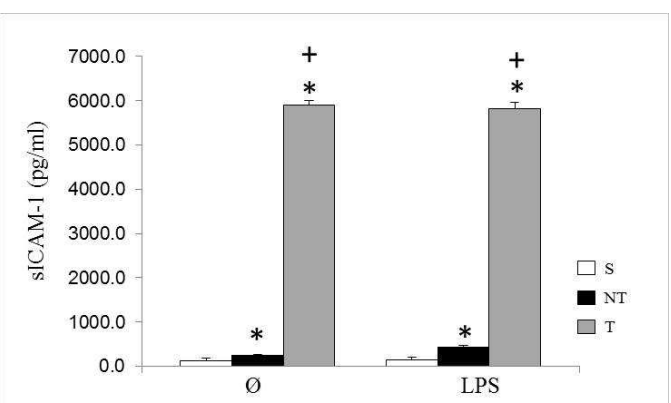

Figure 5. Shedding of ICAM-1 by tested PMF

Concentration of solubile ICAM-1 was measured in cell supernatants by ELISA method according to the manufacturer's instructions

Values are means \pm S.E. $(n=3),{ }^{*} \mathrm{P}<0.05$ vs. $\mathrm{S},+<0.05$ vs. NT

In this research, we wanted to see if application of natural protectors such as water highly enriched in $\mathrm{SiO} 2$ has the ability to recover different PMF functions. Silica is a common component of the diet found mainly in plant-based foods (cereal grains and some fruits and vegetables), drinking water and some alcoholic beverages, notably beer. Currently there are no recommended intakes for dietary silica. Its absorption depends on the food source, with silicon in drinking water being particularly well-absorbed $(>50 \%$ of the total silica content in the food) [4].Our study showed that treatment with water enriched with silicium dioxide in concentration of $20 \mathrm{mg} / \mathrm{L}$ partially normalized characteristic of PMF of animals intoxicated with aluminum. Silicon present in drinking water may be protective with respect to the decrease of cognitive function as it was suggested by several studies [11], [38]. The silica is probably the natural antidote of the aluminum and could play a benefit role by decreasing the toxicity of aluminum [38]. On the other hand, $\mathrm{SiO} 2$ are also associated with silicosis. The main feature of silicosis is high degree of inflammation and substantially scaring in lungs. There are several reports on the pathogenesis of silicosis induced by crystalline silica. Investigators have studied the effects of crystalline silica particles on the expression of cytokines, chemokines, reactive oxygen species (ROS), reactive nitrogen species (RNS), and NO generated mainly through iNOS [39], [40]. Park and Park [29] showed that silica in high concentration $(50,100$, and $250 \mathrm{mg} / \mathrm{kg}$ ) can be toxic to macrophages and other cell types due to high ROS, RNS, and NO effects. However, Arkhipov showed that i.p. injection of $\mathrm{SiO}_{2}$ in the low concentration $(1.7 \mathrm{mg} / \mathrm{ml})$ have no cytotoxic effect on PMF but enhances phagocytosis activity [27]. Probably, we may assume that the effect of silica depends on its form, applied concentration and route of entrance to organism.

Exley group suggest that silicic acid in interaction with Al form hydroxyaluminosilicates, which could explain the influence of silicic acid on the absorption and urinary excretion of $\mathrm{Al}$ [41]. Their further results confirmed that regular drinking of silicic acid-rich potable waters may be an effective mechanism whereby Al might be purged from the body and the body burden of $\mathrm{Al}$ retained at a lowest possible level. [42] This is in accordance with our results where we showed protective role of silica in decreasing the aluminum toxicity.

Finally, results of this study may be explained with the fact that $\mathrm{Al}$ exposure causes immunosuppression, and that $\mathrm{SiO} 2$ in low doses (as it was in our study) may have stimulatory effects which as final outcome has partial restoration of PMF function. Regarding that, our results suggest that silicon dioxide is a natural antidote of the aluminum and we may presume its possible benefit role in decreasing the aluminum toxicity commonly present in water and food.

\section{REFERENCES}

1. K. Michel, J. Scheel, S. Karsten, N. Stelter and T. Wind, "Risk Assessment of Amorphous Silicon Dioxide Nanoparticles in a Glass Cleaner Formulation," Nanotoxicol., vol. 7, no. 5, pp. 974-988, Aug. 2013

2. E.M. Carlisle, "Biochemical and Morphological Changes Associated with Long Bone Abnormalities in Silicon Deficiency," J. Nutr., vol. 110, no. 5, pp.1046-1056, May 1980

3. E.M. Carlisle, "The Nutritional Essentiality of Silicon," Nutr. Rev., vol. 40, no. 7, pp.193-198, July 1982

4. H.M. Macdonald, A.C. Hardcastle, R. Jugdaohsingh, W.D. Fraser, D.M. Reid, J.J. Powell, "Dietary Silicon Interacts With Oestrogen to Influence Bone Health: Evidence from the Aberdeen Prospective Osteoporosis Screening Study," Bone, vol. 50, no. 3, pp. 681-687, Mar. 2012

5. R. Jugdaohsingh et al., "Increased Longitudinal Growth in Rats on a Silicon-Depleted Diet," Bone, vol. 43, no. 3 , pp. 596-6o6, Sep. 2008 
6. A.Becaria, A.Campbell, and S.C. Bondy, "Aluminum as a Toxicant,” Toxicol. Ind. Health, vol. 18, no. 7, pp. 309320, Aug. 2002

7. P. Nayak, "Aluminum: Impacts and Disease," Envir. Res., vol. 89, no. 2, pp. 101-115, June 2002

8. A. Lerner, "Aluminum is a Potential Environmental Factor for Crohn's Disease Induction: Extended Hypothesis," Ann. NY. Acad. Sci. C, vol. 1107, pp. 329345, June 2007

9. G.P. de Chambrun et al., "Aluminum Enhances Inflammation and Decreases Mucosal Healing in Experimental Colitis in Mice," Mucosal. Immunol, vol. 7, no. 3 , pp. 589-601, May 2014

10. F. Mannello, D. Ligi and M. Canale, "Aluminium, Carbonyls and Cytokines in Human Nipple Aspirate Fluids: Possible Relationship between Inflammation, Oxidative Stress and Breast Cancer Microenvironment," J. Inorg. Biochem., vol. 128, pp. 250-256, Nov. 2013

11. V. Rondeau, H. Jacqmin-Gadda, D. Commenges, C. Helmer, and J.-F. Dartigues, "Aluminum and Silica in Drinking Water and the Risk of Alzheimer's Disease or Cognitive Decline: Findings From 15-Year Follow-up of the PAQUID Cohort," Am. J. Epidemiol., vol. 169, no. 4, pp. 489-496, Feb. 2009

12. C. Hu et al., "Effects of Aluminum Exposure on the Adherence, Chemotaxis and Phagocytosis Capacity of Peritoneal Macrophages in Rats," Biol. Trace. Elem. Res, vol. 144, no. 1, pp. 1032-1038, Dec. 2011

13. V. Castranova et al., "Enhancement of Nitric Oxide Production by Pulmonary Cells Following Silica Exposure," Environ. Health. Persp., vol. 106, suppl. 5, pp. 1165-1169, Oct. 1998

14. R.M. Gilberti, G.N. Joshi and D.A. Knecht, "The Phagocytosis of Crystalline Silica Particles by Macrophages," Am. J. Respir. Cell. Mol. Biol., vol. 39, no. 5, pp. 619-627, Nov. 2008

15. H.Y. Chen, I.C. Weng, C.S. Li, L. Wan and F.T. Liu, "Examination of Galectins in Phagocytosis", Meth. Molec. Biol., vol.1207, pp. 201-213, Aug. 2014

16. M. Hashizume and M. Mihara, "Atherogenic Effects of TNF- $\alpha$ and IL-6 via Up-Regulation of Scavenger Receptors," Cytokine, vol. 58, no. 3, pp. 424-430, June 2012

17. A. Solhaug et al., "Autophagy and Senescence, Stress Responses Induced by the DNA-Damaging Mycotoxin Alternariol," Toxicol. Lett., vol. 326, pp. 119-129, Dec. 2014

18. Z. Gao et al., "Effects of Selenizing Angelica Polysaccharide and Selenizing Garlic Polysaccharide on Immune Function of Murine Peritoneal Macrophage," Int. Immunopharmacol., vol. 27, no. 1, pp. 104-109, July 2015

19. A. Dahdah et al., "Mast Cells Aggravate Sepsis By Inhibiting Peritoneal Macrophage Phagocytosis," J. Clin. Invest., vol. 124, no. 10, pp. 4577-4589, Oct. 2014

20. M.C.C. Machado and A.M.M. Coelho, "Role of Peritoneal Macrophages on Local and Systemic Inflammatory Response in Acute Pancreatitis," in Acute Pancreatitis, Luis Rodrigo, Ed., Rijeka, Croatia: InTech, 2012, Ch. 8

21. C.A. Shaw, S. Seneff, S.D. Kette, L. Tomljenovic, J.W. Oller Jr. and R.M. Davidson, "Aluminum-Induced Entropy in Biological Systems: Implications for Neurological Disease," rev. art., J. Toxicol., vol. 2014, Oct. 2014

22. A.C. Rimaniol et al., "Aluminum Hydroxide Adjuvant Induces Macrophage Differentiation Towards a Specialized Antigen-Presenting Cell Type," Vaccine, vol. 22, no. 23-24, pp. 3127-3135, Aug. 2004

23. S.L. Tong, "The Effects of Aluminum, Zinc, Copper, Chromium Ion on the Phagocytosis Capacity of Macrophage in Mice," J. Immunol. Chin., no. 6, pp. 219221, 1990

24. A.J. Wagner, C.A. Bleckmann, R.C. Murdock, A.M Schrand, J.J. Schlager and S.M. Hussain, "In Vitro Toxicity of Aluminum Nanoparticles in Rat Alveolar
Macrophages," J. Phys. Chem. B, vol. 111, no. 25, pp. 7353-7359, 2007

25. N. Goto, H. Kato, J. Maeyama, K. Eto and S. Yoshihara "Studies on the Toxicities of Aluminium Hydroxide and Calcium Phosphate as Immunological Adjuvants for Vaccines," Vaccine, vol. 11, no. 9, pp. 914-918, 1993

26. A. Aderem and D.M. Underhill, "Mechanisms of Phagocytosis in Macrophages," Annu. Rev. Immunol., vol. 17, pp. 593-623, Apr. 1999

27. S.A. Arkhipov, V.A. Shkurupy and Yu.S. Bugrimova, "Effect of Preliminary Load of Macrophages with Silicium Dioxide on Phagocytosis of BCG Strain Micobacteria by Macrophages and Antimicrobial Activity," Bull. Exp. Biol. Med., vol. 149, no. 4, pp. 534536, Oct. 2010

28. T. Röszer, "Understanding the Mysterious M2 Macrophage through Activation Markers and Effector Mechanisms," Mediat. Inflamm., vol. 2015, 2015

29. E.J. Park and K. Park, "Oxidative Stress and ProInflammatory Responses Induced by Silica Nanoparticles In Vivo and In Vitro," Toxicol. Lett., vol. 184, no. 1, pp. 18-25, Jan. 2009

30. A. Deleporte et al., "Efficacy of Anti-TNF-Alpha Monoclonal Antibodies in Inflammatory Bowel Disease Treatment," Int. J. Interferon. Cytokine. Mediat. Res., vol. 5, pp. 11-31, Oct. 2013

31. W. Swardfager, K. Lanctôt, L. Rothenburg, A. Wong, J. Cappell and N. Herrmann, "A Meta-Analysis of Cytokines in Alzheimer's Disease,” Biol. Psychiatry., vol. 68 , no. 10, pp. 930-941, Nov. 2010

32. R.M. Locksley, N. Killeen and M.J. Lenardo, "The TNF and TNF Receptor Superfamilies: Integrating Mammalian Biology," Cell, vol. 104, no. 4, pp. 487-501, Feb. 2001

33. L. Yang, R.M. Froio, T.E. Sciuto, A.M. Dvorak, R. Alon, F.W. Luscinskas, "ICAM-1 Regulates Neutrophil Adhesion and Transcellular Migration of TNF- $\alpha-$ Activated Vascular Endothelium under Flow," Blood, vol. 106, no. 2, pp.584-592, July 2005

34. S. Etienne-Manneville, N. Chaverot, A.D. Strosberg and P.O. Couraud, "ICAM-1-Coupled Signaling Pathways in Astrocytes Converge to Cyclic AMP Response ElementBinding Protein Phosphorylation and TNF-Alpha Secretion,” J. Immunol., vol. 163, no. 2, pp. 668-674, July 1999

35. A.M. Witkowska and M.H. Borawska, "Soluble Intercellular Adhesion Molecule-1 (SICAM-1): an Overview," Eur. Cytokine. Netw., vol. 15, no. 2, pp. 9198, Apr.-June 2004

36. A.K. Hubbard and C. Giardina. "Regulation of ICAM-1 Expression in Mouse Macrophages," Inflammation, vol. 24, no. 2, pp.115-125, Apr. 2000

37. N.G. Plekhova, L.M. Somova, E.I. Drobot, N.V. Krylova and G.N. Leonova, "Changes in the metabolic activity of macrophages under the influence of tick-borne encephalitis virus ", Biochemistry, vol. 72(2), pp. 199207, Feb 2007

38. G.S. Guyonnet, S. Andrieu and B. Vellas, "The Potential Influence of Silica Present in Drinking Water on Alzheimer's Disease and Associated Disorders," J. Nutr. Health. Aging, vol. 11, no. 2, pp. 119-124, Mar.-Apr. 2007

39. B. Rimal, A.K. Greenberg and W.N. Rom, "Basic Pathogenetic Mechanisms in Silicosis: Current Understanding," Curr. Opin. Pulm. Med., vol. 11, no. 2, pp. 169-173, Mar. 2005

40. J. Øvrevik et al., "Mechanisms of Silica-Induced IL-8 Release from A549 Cells: Initial Kinase-Activation does not Require EGFR Activation or Particle Uptake," Toxicol., vol. 227, no. 1-2, pp. 105-116, Oct 2006

41. C. Exley, C. Schneider and F.J. Doucet, "The Reaction of Aluminium with Silicic Acid in Acidic Solution: an Important Mechanism in Controlling the Biological Availability of Aluminium?" Coord. Chem. Rev., vol. 228, no. 2, pp.127-135, June 2002 
42. C. Exley, O. Korchazhkina, D. Job, S. Strekopytov, A. Polwart and P.Crome, "Non-Invasive Therapy to Reduce the Body Burden of Aluminium in Alzheimer's Disease,"

J. Alzheimers. Dis., vol. 10, no. 1, pp.17-24, Sep. 2006 